\title{
Snapping out of Autopilot: Overriding Habits in Real Time and the Role of Ventrolateral Prefrontal Cortex
}

Cole Korponay ${ }^{1,2}$, Ph.D., M.P.A., ckorponay@mclean.harvard.edu

${ }^{1}$ Basic Neuroscience Division, McLean Hospital, Belmont, MA

${ }^{2}$ Department of Psychiatry, Harvard Medical School, Cambridge, MA

*Author for correspondence: Cole Korponay

ckorponay@mclean.harvard.edu

115 Mill St.

Belmont, MA 02478

$\underline{\text { Acknowledgements }}$

Funded by National Institute on Drug Abuse grant 1F32DA048580 to CK 


\section{$\underline{\text { Abstract }}$}

Habits allow environmental and interoceptive cues to trigger behavior in an automatized fashion, making them liable to deployment in inappropriate or outdated contexts. Over the longterm, repeated failure of a once adaptive habit to satisfy current goals produces extinction learning that suppresses the habit's execution. Less attention has been afforded to the mechanisms underlying real-time habit suppression: the capacity to stop the execution of a cued habit that is goal-conflicting. Here, we first posit a model by which goal-relevant stimuli can 1) bring unfolding habits and their projected outcomes into awareness, 2) prompt evaluation of the habit outcome with respect to current goals, and 3) trigger cessation of the habit response if it is determined to be goal-conflicting. Second, we propose a modified stop-signal task to test this model of "goal-directed stopping of habit execution". Finally, we marshal evidence indicating that the ventrolateral prefrontal cortex (vlPFC), situated at the nexus of salience detection, action-plan assessment, and motor inhibition networks, is uniquely positioned to coordinate the overriding of habitual behaviors in real time. In sum, we present a testable model and candidate neurobiological substrate for our capacity to "snap out of autopilot" and override goal-conflicting habits in real time. 


\section{$\underline{\text { Introduction }}$}

Habits develop after taking the same action in a given context consistently leads to reward (Dickinson, Klein, \& Mowrer, 1989; Dolan \& Dayan, 2013; Thorndike, 1927). In such contexts, the need for conscious deliberation about what action to take to achieve the goal eventually becomes unnecessary. When the stimuli associated with these contexts present themselves, they automatically trigger the action that has repeatedly yielded reward in that context. The gradual transfer of such behaviors from the control of a deliberative, "goaldirected" behavior system to a reflexive, "habit-based" behavior system facilitates metabolic efficiency and frees up cognitive resources to engage elsewhere (Robbins \& Costa, 2017). A rich literature has detailed the neural processes devoted to this transfer mechanism, wherein frequently repeated goal-directed behaviors encoded in dorsomedial striatum are shuttled and reconfigured via the dopaminergic striatonigrostriatal pathways (Belin \& Everitt, 2008; Haber, Fudge, \& McFarland, 2000) to storage as stimulus-response habits in dorsolateral striatum (Barry J Everitt \& Robbins, 2005, 2016).

The downside to habits is that what is gained in efficiency is lost in flexibility. Habits are generally insensitive to changes in current goals, outcome values and contingencies (Dickinson, 1994; Robbins \& Costa, 2017). Therefore, when a habit-cueing stimulus takes place amidst a larger context where goals may be different than usual, habits can lead us astray. "Have a great flight!" says the airport check-in employee after you are done checking in. "You too!" you say back instinctively. The first intersection after leaving home comes up, and you make the usual left turn toward work despite being on your way to the doctor's office. You walk out of the bathroom and turn the lights off, even though your spouse is still in there brushing their teeth. These instances of "habitual action-slips" (Gillan et al., 2011; Norman, 1981) underscore the 
potential for usually goal-satisfying habits to deploy in inappropriate contexts that undercut our goals. While the action-slips described here may amount to minor nuisances, action-slips occur with greater frequency in certain neuropsychiatric disorders like obsessive-compulsive disorder (Gillan et al., 2011) that can contribute to severe functional impediments.

In healthy individuals, the repeated failure of a once adaptive habit to satisfy the goals of a changed environment eventually results in extinction learning that suppresses the habit's execution (Bouton, 2004; Goodman, Ressler, \& Packard, 2016; Rescorla, 2001). But what if we need to halt an active/non-extinguished habit in real time? Unlike physiological reflexes that bypass the brain, habits are not inescapably executed to completion upon the incidence of their initiating stimuli (Pollard, 2006). A shared human experience is the phenomenon of suddenly catching ourselves amidst some seemingly autopiloted action - often which we did not consciously initiate - that is leading us astray from our current goal and changing course. What are the mechanisms that facilitate this "snapping out of autopilot" capacity?

In the following sections, we formalize a model for overriding habits in real time, propose a task-based means for evaluating this model, and review evidence that points to the ventrolateral prefrontal cortex as a crucial brain region for this capacity.

\section{A Model of Overriding Habits in Real Time}

Traditional conceptualizations of goal-directed and habitual behavior posit that these are two independent and non-interacting systems that govern separate aspects of behavior (Dickinson, 1985). After all, habits, by most definitions, are stimulus-triggered actions that bypass considerations of goals and outcomes. However, recent work has increasingly recognized the interplay between the goal-directed (model-based) and habit (model-free) systems at the computational, neural and behavioral levels (Daw, 2018; Keramati, Smittenaar, Dolan, \& Dayan, 
2016; Krueger \& Griffiths, 2018). Along these lines, we posit a model of "goal-directed stopping of goal-conflicting habits" that entails interaction between the goal-directed and habitual behavior systems.

After an initial stimulus/cue (Figure 1a) has begun to initiate a habit response (Figure 1b), the process of overriding the execution of that habit in real time requires several sequential steps. First, there must be subsequent perception of a goal-relevant stimulus/cue (Figure 1c). As a default, stimulus-response habit cascades occur beneath the level of awareness; this secondary, goal-relevant stimulus "snaps the system out of autopilot" and brings the unfolding habit into awareness (Figure 1d). This then prompts an evaluation of the habit response's prospective outcome in the context of current goals (Figure 1e). This entails a direct comparison of the outcome utility to be provided by executing the habit and that to be provided by taking an alternative, goal-directed action. If the result of this evaluation is a determination that the habit's execution will conflict with current goals, then the third step is inhibition or cessation of the habit response and execution of the alternate, goal-directed action (Figure 1f).

\section{Testing the Model}

\section{Motivating a Task Design}

The laboratory task that comes closest to modeling the overriding of a habit in real time is the stop-signal task (Li, Huang, Constable, \& Sinha, 2006; Logan, Cowan, \& Davis, 1984). While subjects are not tasked to override a habit per se, they are tasked to cease the execution of an already initiated, prepotent, stimulus-response cascade. At a minimum, the stop-signal task demonstrates that even amidst an already initiated stimulus-response cascade, the perception of a secondary stimulus can prompt the cancellation of this cascade when its outcome conflicts with a newly current goal signaled by the secondary stimulus (Verbruggen et al., 2019). 
In the traditional stop-signal task, however, the secondary "stop" signal likely does not promote stopping by prompting awareness of the initial stimulus-response cascade and facilitating consideration of its outcome relative to current goals. Rather, it initiates a parallel, competing action (to halt movement), via the "hyperdirect" cortical-subthalamic nucleus pathway that cancels ongoing movements (Adam R Aron, Behrens, Smith, Frank, \& Poldrack, 2007; Chen et al., 2020). In this way, stopping in the traditional stop-signal task is reflexive rather than goal-directed. To prompt and evaluate "goal-directed stopping" of a habit that is recognized to be goal-conflicting, we propose several modifications to the traditional stop signal task.

In order to probe goal-directed stopping and not reflexive stopping, the secondary "stopsignal" stimulus must be voided of its automatic association with stopping. That is, the subject must not be explicitly trained to inhibit behavior in response to the presentation of a secondary stimulus as a default action. A variation of the traditional stop-signal task, the "conditional" stopsignal task (Adam R Aron et al., 2007; Obeso et al., 2011), achieves this by presenting one of two types of primary "go" stimuli before the presentation of the secondary "stop" stimulus: a "critical" primary stimulus or a "non-critical" primary stimulus. Presentation of the "noncritical" primary stimulus (e.g., a left-pointing arrow) instructs the subject not to inhibit the "go" response even if the secondary "stop" stimulus is subsequently presented. Alternatively, presentation of the "critical" primary stimulus (e.g., a right-pointing arrow) instructs the subject to inhibit the "go" response if the secondary "stop" stimulus is subsequently presented. However, despite voiding the secondary stimulus of an automatic mandate to stop, doing so by imbuing the primary habit-cueing "go" stimulus with a prescribed conditional instruction does not test the capacity and model of "snapping out of autopilot" that is of interest here. First, this manipulation 
arguably prevents the formation of a strong prepotent response in relation to the primary stimulus, since the subject must engage active attention both during the presentation of the primary stimulus (to identify whether it is "critical" or "non-critical" and to retrieve the appropriate response contingency) and after it (to maintain the appropriate response contingency in working memory until the secondary stimulus is or is not presented). Second, it does not provide a way to examine whether the nature of the secondary stimulus (i.e., goal-relevant versus goal-irrelevant; goal-conflicting versus goal-consistent) modulates the extent to which an ongoing habit response can be recognized and adjusted in a goal-directed manner. Accomplishing this requires the presentation of varied secondary stimuli, for which it is the subject's prerogative to determine - in a goal-directed manner - if task conditions and current goals warrant stopping. Therefore, in order to 1) void the secondary stimulus of its automatic association with stopping, 2) promote the formation of a strong prepotent response to the primary stimulus, and 3) test the capacity of different classes of secondary stimuli to "snap the system out of autopilot", the following variation of the stop signal task is proposed.

\section{A “Goal-Directed” Stop-Signal Task}

First, a primary stimulus is presented - a dollar amount that decreases rapidly every millisecond that the subject hasn't pressed the "take" keyboard button. Then, on $70 \%$ of trials ("goal-irrelevant" trials), a set of three random (non-monetary) secondary stimuli are also presented quickly after initial stimulus onset. This structure builds up a prepotent response to press the take button as quickly as possible upon initial stimulus presentation, even as secondary stimuli are presented as well. On 30\% of trials ("goal-relevant" trials), one of the secondary stimuli is another dollar amount. In half of these instances ("goal-relevant, goal-consistent" trials), the amount is significantly smaller than the original amount, in which the optimal subject 
action is still to press the "take" button. In the other half of these instances ("goal-relevant, goalconflicting" trials), the amount is significantly larger than the original amount, in which the optimal subject action is to inhibit pressing the take button. This structure allows subjects to determine, in a goal-directed manner, which secondary stimuli warrant overriding the habitual button-press response.

This task structure is thereby suited to examine the capacity of goal-relevant stimuli to prompt recognition of an ongoing habit, and the capacity of goal-relevant stimuli that are also goal-conflicting to stop the habit's execution. In doing so, the task allows measurement of subject variability in the three component aspects that our model proposes are necessary for goaldirected stopping of goal-conflicting habits: 1) reflexive reorienting to goal-relevant stimuli (increase in reaction time on "goal-relevant" compared to "goal-irrelevant" trials), comparative evaluation of the utility of the habit response outcome versus the goal-directed stopping outcome (accuracy on "goal-relevant" trials), and motor inhibition (the speed of stopping on "goalrelevant, goal-conflicting” trials).

Other variations of the stop-signal task have shown that leveraging environmentally salient stimuli (e.g., red and green traffic lights), which already have habitual go/stop responses associated with them, can be an effective means to ensure the examination of a genuine habitual behavior on the task (Ceceli, Myers, \& Tricomi, 2020; Hochman, Henik, \& Kalanthroff, 2018). Moreover, tasks of this nature have shown that monetary incentives - when combined with realtime feedback - can weaken habitual behaviors (Ceceli et al., 2020). Thus, to parse out potential influences of habit strength and monetary reward on the "snapping out of autopilot" capacity, it may be of interest to compare performance on the version of the task proposed here (which uses monetary-stimuli and performance-linked monetary rewards) with performance on versions that 
instead use environmentally salient stimuli and/or do not use performance-linked monetary rewards. Furthermore, it may also be useful to include a habit-check component that indexes the strength of habit formation for each subject. This could include, on a small subset of trials, having the primary stimulus increase in monetary value over time, in which case optimal subject behavior is to withhold pressing the "take button". Insensitivity to this change in outcome gauged by each subject's average payout on such trials - could provide a useful index of habit strength.

\section{Role of the Ventrolateral Prefrontal Cortex}

The ventrolateral prefrontal (vlPFC) is a strong candidate substrate for orchestrating the component processes required to override a goal-conflicting habit in real time. In the human, the vlPFC occupies the inferior frontal gyrus (IFG) of the frontal lobe and consists of three broad anatomical and functional subregions: the pars orbitalis (Brodmann areas 47), the pars triangularis (Brodmann area 45), and the pars opercularis (Brodmann area 44). These vlPFC subregions have been variously implicated in salience detection, outcome evaluation, action-plan switching, arbitrating between the execution of habitual and goal-directed behavior, and motor inhibition. Importantly, structural connections link each of these subregions to each other (M. Petrides \& D. N. Pandya, 2002; K. S. Saleem, B. Miller, \& J. L. Price, 2014), allowing for the transfer of information between the vlPFC's functional subunits. Below, we review the evidence for the vlPFC's roles in these functions and the structural and functional connections that facilitate them (Figure 2).

\section{Salience Detection and Reflexive Reorienting}

The vlPFC is a primary node in both the salience network $(\mathrm{SN})$ - which also includes the adjacent anterior insula and the dorsal anterior cingulate cortex (dACC) (Seeley et al., 2007) - 
and the bottom-up fronto-parietal-temporal ventral attention network (VAN) (Corbetta, Patel, \& Shulman, 2008). The SN and VAN are central to rapid detection of and orienting attention toward goal-relevant stimuli. The role of vlPFC in these processes is largely facilitated by direct inputs received from higher-order sensory processing areas and from the amygdala. These connections, delineated by non-human primate tract-tracing studies, are detailed below. Visual Input. The vlPFC receives direct inputs from extrastriate visual cortical areas in the inferotemporal cortex via the uncinate fasciculus, (Bullier, Schall, \& Morel, 1996; Ungerleider, Gaffan, \& Pelak, 1989). These connections help form the ventral visual "what" stream that processes object identity (Mishkin, Ungerleider, \& Macko, 1983). vlPFC neurons in areas 12/47 and 45 that are innervated by these inferotemporal visual areas have been shown to be face and object selective (Scalaidhe, Wilson, \& Goldman-Rakic, 1997; Wilson, Scalaidhe, \& GoldmanRakic, 1993).

Auditory Input. Similarly, vlPFC receives input from auditory processing regions of the superior temporal cortex. vIPFC areas $12 / 47$ and 45 receive input from the lateral auditory belt, which processes complex auditory stimuli such as species-specific vocalizations and band-passed noise (Romanski et al., 1999). This has led to the supposition that, similar to its role for visual stimuli, vlPFC is involved in identification of auditory stimuli (Romanski et al., 1999). Indeed, individual vlPFC neurons have been shown to integrate both auditory and visual information of this kind (Sugihara, Diltz, Averbeck, \& Romanski, 2006).

Amygdalar Input. The same vlPFC subregions that receive processed visual and auditory input are also reciprocally connected to the amygdala. Areas 45A and 45B in particular have amygdalar connections that are markedly richer than those of surrounding areas (Gerbella, Baccarini, Borra, Rozzi, \& Luppino, 2014). It is thought that these connections may imbue the 
sensory input that vIPFC receives with value or emotional valence, in order to guide visual gaze and attention toward salient and behaviorally relevant visual and auditory stimuli (Adolphs, 2010; Gerbella et al., 2014).

Overall, the higher-order sensory and amygdalar connections of the vlPFC provide it with direct access to the type of information needed to recognize and reorient attention toward stimuli that are salient or goal-relevant. It is postulated that these information streams ultimately contribute to the formation of "priority maps" in the vlPFC that guide attention to the most behaviorally relevant stimuli (Gerbella et al., 2014).

\section{Evaluating Action Plan Alternatives}

Converging evidence from human fMRI and animal models has long pointed to distinct corticostriatal circuits for the processing of goal-directed behavior (i.e., dorsolateral prefrontal cortex, ventromedial prefrontal cortex, orbitofrontal cortex and the caudate) and habitual behavior (i.e., premotor cortex, supplementary motor areas, and the putamen) (Balleine \&

O'doherty, 2010; H. H. Yin \& B. J. Knowlton, 2006). The prospective planning functions of dIPFC and adaptable action-value representations in vmPFC and OFC help to facilitate flexible goal-directed behavior, while action sequences and stimulus-responses associations stored in the motor cortical areas and putamen drive efficient habitual behaviors (Balleine \& O'doherty, 2010; de Wit et al., 2012; McNamee, Liljeholm, Zika, \& O'Doherty, 2015; Smittenaar, FitzGerald, Romei, Wright, \& Dolan, 2013; Tricomi, Balleine, \& O’Doherty, 2009; Valentin, Dickinson, \& O'Doherty, 2007; van Steenbergen, Watson, Wiers, Hommel, \& de Wit, 2017; Henry H Y in \& Barbara J Knowlton, 2006). Non-human primate tract-tracing studies demonstrate that the vlPFC is connected to the cortical components (M. Petrides \& D. Pandya, 2002; Kadharbatcha S Saleem, Brad Miller, \& Joseph L Price, 2014) and striatal components (Ferry, Ongur, An, \& 
Price, 2000; Korponay, Choi, \& Haber, 2020) of both circuits. Moreover, vlPFC has recently been linked in human fMRI studies to facilitating comparative evaluations of goal-directed and habitual actions, and arbitrating which system predominates in a given context (Gruner, Anticevic, Lee, \& Pittenger, 2016; Lee, Shimojo, \& O'Doherty, 2014).

Early fMRI studies highlighted that activity in the vlPFC accompanied behavioral switching on reversal learning tasks (Cools, Clark, Owen, \& Robbins, 2002) and the cessation of habitual behavior on go/no-go tasks (Garavan, Ross, \& Stein, 1999). More recently, a computational fMRI study (Lee et al., 2014) in humans used a sequential Markov decision task, which differentially favored either goal-directed (model-based) or habitual (model free) control of behavior on a given trial, to assess where the brain arbitrates the reliability of each behavioral system. It found that bilateral vlPFC activity encoded both goal-directed and habitual reliability signals as well as, importantly, the output of a comparison between those two signals (a maximum reliability signal). In other words, vlPFC activity correlated with whichever system made the better prediction on a given trial. Furthermore, functional connectivity between the vlPFC and the posterior putamen and SMA (regions underlying habitual behavior) was modulated by the maximum reliability signal in the vlPFC: the more the maximum reliability signal in vlPFC favored goal-directed control of behavior, the more negative coupling there was between the vIPFC and the habitual behavior regions. In a separate study, obsessive-compulsive disorder (OCD) patients, who exhibit imbalance between goal-directed and habitual behavior, were found to have reduced global functional connectivity in the same region of vlPFC (Anticevic et al., 2014), and another study in OCD patients found reduced functional connectivity between the vIPFC and caudate that was associated with poor cognitive flexibility (Vaghi et al., 2017). Furthermore, in healthy human subjects, striatal dopamine levels associated 
with goal-directed (model-based) behavior have been found to accompany goal-directed encoding in the vlPFC (Deserno et al., 2015), suggestive of a possible link between the vlPFC and the dopamine-driven dorsomedial to dorsolateral striatal shifts (B. J. Everitt \& Robbins, 2013). Most recently, transcranial direct current stimulation applied to the vlPFC was found to causally modulate the favoring of either model-free (habit) or model-based (goal-directed) learning systems (Weissengruber, Lee, O’Doherty, \& Ruff, 2019).

Collectively, these findings implicate the vlPFC as a central cortical component in evaluating action plans and arbitrating between the execution of goal-directed or habitual behavior depending on current goals.

\section{Inhibition}

The vlPFC is known to play a role in inhibition across numerous domains, including the inhibition of actions, memories, thoughts and emotions (Dillon \& Pizzagalli, 2007; Hooker \& Knight, 2006). Converging findings from fMRI, lesion, and transcranial magnetic stimulation (TMS) studies point to vlPFC area 44, corresponding to the pars opercularis of the inferior frontal gyrus in humans, as the vlPFC subregion most consistently linked to motor inhibition (A. R. Aron, Robbins, \& Poldrack, 2014). The structural connections of vlPFC area 44 strongly support its role inhibitory functions. For instance, non-human primate tract-tracing studies show that vlPFC area 44 receives strong input from premotor cortex (Frey, Mackey, \& Petrides, 2014), and corticostriatal projections from area 44 converge with those from premotor cortex in the ventrolateral putamen (Korponay et al., 2020). fMRI studies in humans of motor inhibition across a range of tasks find that these three regions - vlPFC area 44, premotor cortex, and ventrolateral putamen - are consistently activated in relation to motor inhibition (Jahanshahi, Obeso, Rothwell, \& Obeso, 2015; Majid, Cai, Corey-Bloom, \& Aron, 2013; Schel, 
Ridderinkhof, \& Crone, 2014; Simmonds, Pekar, \& Mostofsky, 2008; Zandbelt \& Vink, 2010). For example, one study found that ventrolateral putamen activity that co-occurred with vlPFC and SMA activity was associated with reduced activity in motor cortex and corresponded with successful inhibition of motor activity on the stop-signal task (Zandbelt \& Vink, 2010). Another study found a similar compilation of dorsal caudal vlPFC activity, preSMA activity, and ventrolateral putamen activity during volitional stopping on the marble task (Schel et al., 2014). Furthermore, a meta-analysis of studies using the go/no-go task found that vlPFC area 44, preSMA area $6 / 32$, premotor cortex area 6 , and putamen were among the regions consistently activated during successful inhibition on no-go trials (Simmonds et al., 2008).

Overall, the structural and functional circuitry of the vlPFC place it at the nexus of salience detection, action plan evaluation, and inhibition networks - the three functions proposed as necessary for overriding habits in real time. In this way, the vlPFC is equipped to alert us to the presence of goal-relevant stimuli, evaluate whether continued execution of the habit or switching to an alternative goal-directed action aligns better with current goals, and inhibit execution of the habit if it is determined to be goal-conflicting. Signatures of vlPFC activity during these stages of the modified stop-signal task would help to bolster this account.

\section{$\underline{\text { Conclusion }}$}

The brain has a well-established, long-term mechanism - extinction learning - for decreasing the deployment of no longer adaptive habits over time. The process by which the brain temporarily halts goal-conflicting habits in real time has received less examination. Here, we have reviewed evidence of the brain's capacity for this "snapping out of autopilot" function, posited a model through which the brain implements this function, proposed a task to test and assess the model, and marshaled evidence in support of the central role of the vlPFC in this 
function. Collectively, this framework provides a jumping off point for future research into the brain's capacity for goal-directed stopping of habits in real time. For example, examination of aberrance in this function may be warranted in forms of psychopathology that feature deficits in reinforcement learning, behavioral flexibility, and/or balancing goal-directed and habitual behavior, such as substance use disorder (SUD) (McKim, Bauer, \& Boettiger, 2016), OCD (Gillan et al., 2011) and action disorganization syndrome (ADS) (Niki, Kumada, Maruyama, Tamura, \& Muragaki, 2019). 


\section{$\underline{\text { References }}$}

Adolphs, R. (2010). What does the amygdala contribute to social cognition? Annals of the New York Academy of Sciences, 1191(1), 42.

Anticevic, A., Hu, S., Zhang, S., Savic, A., Billingslea, E., Wasylink, S., . . Pittenger, C. (2014). Global resting-state functional magnetic resonance imaging analysis identifies frontal cortex, striatal, and cerebellar dysconnectivity in obsessive-compulsive disorder. Biol Psychiatry, 75(8), 595-605. doi:10.1016/j.biopsych.2013.10.021

Aron, A. R., Behrens, T. E., Smith, S., Frank, M. J., \& Poldrack, R. A. (2007). Triangulating a cognitive control network using diffusion-weighted magnetic resonance imaging (MRI) and functional MRI. Journal of Neuroscience, 27(14), 3743-3752.

Aron, A. R., Robbins, T. W., \& Poldrack, R. A. (2014). Inhibition and the right inferior frontal cortex: one decade on. Trends Cogn Sci, 18(4), 177-185. doi:10.1016/j.tics.2013.12.003

Balleine, B. W., \& O'doherty, J. P. (2010). Human and rodent homologies in action control: corticostriatal determinants of goal-directed and habitual action. Neuropsychopharmacology, 35(1), 48-69.

Belin, D., \& Everitt, B. J. (2008). Cocaine seeking habits depend upon dopamine-dependent serial connectivity linking the ventral with the dorsal striatum. Neuron, 57(3), 432-441.

Bouton, M. E. (2004). Context and behavioral processes in extinction. Learning \& memory, 11(5), 485-494.

Bullier, J., Schall, J. D., \& Morel, A. (1996). Functional streams in occipito-frontal connections in the monkey. Behavioural brain research, 76(1-2), 89-97.

Ceceli, A. O., Myers, C. E., \& Tricomi, E. (2020). Demonstrating and disrupting well-learned habits. PloS one, 15(6), e0234424.

Chen, W., de Hemptinne, C., Miller, A. M., Leibbrand, M., Little, S. J., Lim, D. A., . . Starr, P. A. (2020). Prefrontal-subthalamic hyperdirect pathway modulates movement inhibition in humans. Neuron, 106(4), 579-588. e573.

Cools, R., Clark, L., Owen, A. M., \& Robbins, T. W. (2002). Defining the neural mechanisms of probabilistic reversal learning using event-related functional magnetic resonance imaging. J Neurosci, 22(11), 4563-4567. doi:20026435

Corbetta, M., Patel, G., \& Shulman, G. L. (2008). The reorienting system of the human brain: from environment to theory of mind. Neuron, 58(3), 306-324.

Daw, N. D. (2018). Are we of two minds? Nature neuroscience, 21(11), 1497-1499.

de Wit, S., Watson, P., Harsay, H. A., Cohen, M. X., van de Vijver, I., \& Ridderinkhof, K. R. (2012). Corticostriatal connectivity underlies individual differences in the balance between habitual and goal-directed action control. Journal of Neuroscience, 32(35), 12066-12075.

Deserno, L., Huys, Q. J., Boehme, R., Buchert, R., Heinze, H. J., Grace, A. A., . . Schlagenhauf, F. (2015). Ventral striatal dopamine reflects behavioral and neural signatures of modelbased control during sequential decision making. Proc Natl Acad Sci U S A, 112(5), 1595-1600. doi:10.1073/pnas. 1417219112

Dickinson, A. (1985). Actions and habits: the development of behavioural autonomy. Philosophical Transactions of the Royal Society of London. B, Biological Sciences, 308(1135), 67-78.

Dickinson, A. (1994). Instrumental conditioning. Animal learning and cognition, 45-79. 
Dickinson, A., Klein, S., \& Mowrer, R. (1989). Expectancy theory in animal conditioning.

Dillon, D. G., \& Pizzagalli, D. A. (2007). Inhibition of action, thought, and emotion: a selective neurobiological review. Applied and Preventive Psychology, 12(3), 99-114.

Dolan, R. J., \& Dayan, P. (2013). Goals and habits in the brain. Neuron, 80(2), 312-325.

Everitt, B. J., \& Robbins, T. W. (2005). Neural systems of reinforcement for drug addiction: from actions to habits to compulsion. Nature neuroscience, 8(11), 1481-1489.

Everitt, B. J., \& Robbins, T. W. (2013). From the ventral to the dorsal striatum: devolving views of their roles in drug addiction. Neuroscience \& Biobehavioral Reviews, 37(9), 19461954.

Everitt, B. J., \& Robbins, T. W. (2016). Drug addiction: updating actions to habits to compulsions ten years on. Annual review of psychology, 67, 23-50.

Ferry, A. T., Ongur, D., An, X., \& Price, J. L. (2000). Prefrontal cortical projections to the striatum in macaque monkeys: evidence for an organization related to prefrontal networks. J Comp Neurol, 425(3), 447-470. doi:10.1002/10969861(20000925)425:3<447::aid-cne9>3.0.co;2-v

Frey, S., Mackey, S., \& Petrides, M. (2014). Cortico-cortical connections of areas 44 and $45 B$ in the macaque monkey. Brain Lang, 131, 36-55. doi:10.1016/j.band1.2013.05.005

Garavan, H., Ross, T. J., \& Stein, E. A. (1999). Right hemispheric dominance of inhibitory control: an event-related functional MRI study. Proc Natl Acad Sci U S A, 96(14), 83018306. doi:10.1073/pnas.96.14.8301

Gerbella, M., Baccarini, M., Borra, E., Rozzi, S., \& Luppino, G. (2014). Amygdalar connections of the macaque areas 45A and 45B. Brain Structure and Function, 219(3), 831-842.

Gillan, C. M., Papmeyer, M., Morein-Zamir, S., Sahakian, B. J., Fineberg, N. A., Robbins, T. W., \& de Wit, S. (2011). Disruption in the balance between goal-directed behavior and habit learning in obsessive-compulsive disorder. American Journal of Psychiatry, 168(7), 718-726.

Goodman, J., Ressler, R. L., \& Packard, M. G. (2016). The dorsolateral striatum selectively mediates extinction of habit memory. Neurobiol Learn Mem, 136, 54-62. doi:10.1016/j.nlm.2016.09.012

Gruner, P., Anticevic, A., Lee, D., \& Pittenger, C. (2016). Arbitration between Action Strategies in Obsessive-Compulsive Disorder. Neuroscientist, 22(2), 188-198. doi:10.1177/1073858414568317

Haber, S. N., Fudge, J. L., \& McFarland, N. R. (2000). Striatonigrostriatal pathways in primates form an ascending spiral from the shell to the dorsolateral striatum. Journal of Neuroscience, 20(6), 2369-2382.

Hochman, S., Henik, A., \& Kalanthroff, E. (2018). Stopping at a red light: Recruitment of inhibitory control by environmental cues. PloS one, 13(5), e0196199.

Hooker, C. I., \& Knight, R. T. (2006). The role of lateral orbitofrontal cortex in the inhibitory control of emotion. The orbitofrontal cortex, 307.

Jahanshahi, M., Obeso, I., Rothwell, J. C., \& Obeso, J. A. (2015). A fronto-striato-subthalamicpallidal network for goal-directed and habitual inhibition. Nat Rev Neurosci, 16(12), 719732. doi:10.1038/nrn4038

Keramati, M., Smittenaar, P., Dolan, R. J., \& Dayan, P. (2016). Adaptive integration of habits into depth-limited planning defines a habitual-goal-directed spectrum. Proceedings of the National Academy of Sciences, 113(45), 12868-12873. 
Korponay, C., Choi, E. Y., \& Haber, S. N. (2020). Corticostriatal Projections of Macaque Area 44. Cereb Cortex Commun, 1(1), tgaa079. doi:10.1093/texcom/tgaa079

Krueger, P. M., \& Griffiths, T. (2018). Shaping Model-Free Habits with Model-Based Goals. Paper presented at the CogSci.

Lee, S. W., Shimojo, S., \& O'Doherty, J. P. (2014). Neural computations underlying arbitration between model-based and model-free learning. Neuron, 81(3), 687-699. doi:10.1016/j.neuron.2013.11.028

Li, C.-s. R., Huang, C., Constable, R. T., \& Sinha, R. (2006). Imaging response inhibition in a stop-signal task: neural correlates independent of signal monitoring and post-response processing. Journal of Neuroscience, 26(1), 186-192.

Logan, G. D., Cowan, W. B., \& Davis, K. A. (1984). On the ability to inhibit simple and choice reaction time responses: a model and a method. Journal of experimental psychology: human perception and performance, 10(2), 276.

Majid, D. S., Cai, W., Corey-Bloom, J., \& Aron, A. R. (2013). Proactive selective response suppression is implemented via the basal ganglia. J Neurosci, 33(33), 13259-13269. doi:10.1523/JNEUROSCI.5651-12.2013

McKim, T. H., Bauer, D. J., \& Boettiger, C. A. (2016). Addiction history associates with the propensity to form habits. Journal of cognitive neuroscience, 28(7), 1024-1038.

McNamee, D., Liljeholm, M., Zika, O., \& O'Doherty, J. P. (2015). Characterizing the associative content of brain structures involved in habitual and goal-directed actions in humans: a multivariate FMRI study. Journal of Neuroscience, 35(9), 3764-3771.

Mishkin, M., Ungerleider, L. G., \& Macko, K. A. (1983). Object vision and spatial vision: two cortical pathways. Trends in Neurosciences, 6, 414-417.

Niki, C., Kumada, T., Maruyama, T., Tamura, M., \& Muragaki, Y. (2019). Role of Frontal Functions in Executing Routine Sequential Tasks. Front Psychol, 10, 169. doi:10.3389/fpsyg.2019.00169

Norman, D. A. (1981). Categorization of action slips. Psychological review, 88(1), 1.

Obeso, I., Wilkinson, L., Casabona, E., Bringas, M. L., Alvarez, M., Alvarez, L., . . Obeso, J. A. (2011). Deficits in inhibitory control and conflict resolution on cognitive and motor tasks in Parkinson's disease. Experimental brain research, 212(3), 371-384.

Petrides, M., \& Pandya, D. (2002). Comparative cytoarchitectonic analysis of the human and the macaque ventrolateral prefrontal cortex and corticocortical connection patterns in the monkey. European Journal of Neuroscience, 16(2), 291-310.

Petrides, M., \& Pandya, D. N. (2002). Comparative cytoarchitectonic analysis of the human and the macaque ventrolateral prefrontal cortex and corticocortical connection patterns in the monkey. Eur J Neurosci, 16(2), 291-310. doi:10.1046/j.1460-9568.2001.02090.x

Pollard, B. (2006). Explaining actions with habits. American Philosophical Quarterly, 43(1), $57-$ 69.

Rescorla, R. A. (2001). Experimental extinction. Handbook of contemporary learning theories, $55,119-154$.

Robbins, T., \& Costa, R. M. (2017). Habits. Current biology, 27(22), R1200-R1206.

Romanski, L. M., Tian, B., Fritz, J., Mishkin, M., Goldman-Rakic, P. S., \& Rauschecker, J. P. (1999). Dual streams of auditory afferents target multiple domains in the primate prefrontal cortex. Nature neuroscience, 2(12), 1131-1136. 
Saleem, K. S., Miller, B., \& Price, J. L. (2014). Subdivisions and connectional networks of the lateral prefrontal cortex in the macaque monkey. J Comp Neurol, 522(7), 1641-1690. doi:10.1002/cne. 23498

Saleem, K. S., Miller, B., \& Price, J. L. (2014). Subdivisions and connectional networks of the lateral prefrontal cortex in the macaque monkey. Journal of Comparative Neurology, 522(7), 1641-1690.

Scalaidhe, S. P. Ó., Wilson, F. A., \& Goldman-Rakic, P. S. (1997). Areal segregation of faceprocessing neurons in prefrontal cortex. Science, 278(5340), 1135-1138.

Schel, M. A., Ridderinkhof, K. R., \& Crone, E. A. (2014). Choosing not to act: Neural bases of the development of intentional inhibition. Developmental cognitive neuroscience, 10, 93103.

Seeley, W. W., Menon, V., Schatzberg, A. F., Keller, J., Glover, G. H., Kenna, H., . . Greicius, M. D. (2007). Dissociable intrinsic connectivity networks for salience processing and executive control. Journal of Neuroscience, 27(9), 2349-2356.

Simmonds, D. J., Pekar, J. J., \& Mostofsky, S. H. (2008). Meta-analysis of Go/No-go tasks demonstrating that fMRI activation associated with response inhibition is task-dependent. Neuropsychologia, 46(1), 224-232.

Smittenaar, P., FitzGerald, T. H., Romei, V., Wright, N. D., \& Dolan, R. J. (2013). Disruption of dorsolateral prefrontal cortex decreases model-based in favor of model-free control in humans. Neuron, 80(4), 914-919.

Sugihara, T., Diltz, M. D., Averbeck, B. B., \& Romanski, L. M. (2006). Integration of auditory and visual communication information in the primate ventrolateral prefrontal cortex. Journal of Neuroscience, 26(43), 11138-11147.

Thorndike, E. L. (1927). The law of effect. The American journal of psychology, 39(1/4), 212222.

Tricomi, E., Balleine, B. W., \& O’Doherty, J. P. (2009). A specific role for posterior dorsolateral striatum in human habit learning. European Journal of Neuroscience, 29(11), 2225-2232.

Ungerleider, L., Gaffan, D., \& Pelak, V. (1989). Projections from inferior temporal cortex to prefrontal cortex via the uncinate fascicle in rhesus monkeys. Experimental brain research, 76(3), 473-484.

Vaghi, M. M., Vertes, P. E., Kitzbichler, M. G., Apergis-Schoute, A. M., van der Flier, F. E., Fineberg, N. A., . . Robbins, T. W. (2017). Specific Frontostriatal Circuits for Impaired Cognitive Flexibility and Goal-Directed Planning in Obsessive-Compulsive Disorder: Evidence From Resting-State Functional Connectivity. Biol Psychiatry, 81(8), 708-717. doi:10.1016/j.biopsych.2016.08.009

Valentin, V. V., Dickinson, A., \& O'Doherty, J. P. (2007). Determining the neural substrates of goal-directed learning in the human brain. Journal of Neuroscience, 27(15), 4019-4026.

van Steenbergen, H., Watson, P., Wiers, R. W., Hommel, B., \& de Wit, S. (2017). Dissociable corticostriatal circuits underlie goal-directed vs. Cue-elicited habitual food seeking after satiation: Evidence from a multimodal MRI study. European Journal of Neuroscience, 46(2), 1815-1827.

Verbruggen, F., Aron, A. R., Band, G. P., Beste, C., Bissett, P. G., Brockett, A. T., . . Colonius, H. (2019). A consensus guide to capturing the ability to inhibit actions and impulsive behaviors in the stop-signal task. Elife, 8, e46323. 
Weissengruber, S., Lee, S. W., O’Doherty, J. P., \& Ruff, C. C. (2019). Neurostimulation reveals context-dependent arbitration between model-based and model-free reinforcement learning. Cerebral Cortex, 29(11), 4850-4862.

Wilson, F. A., Scalaidhe, S., \& Goldman-Rakic, P. S. (1993). Dissociation of object and spatial processing domains in primate prefrontal cortex. Science, 260(5116), 1955-1958.

Yin, H. H., \& Knowlton, B. J. (2006). The role of the basal ganglia in habit formation. Nat Rev Neurosci, 7(6), 464-476. doi:10.1038/nrn1919

Yin, H. H., \& Knowlton, B. J. (2006). The role of the basal ganglia in habit formation. Nature Reviews Neuroscience, 7(6), 464-476.

Zandbelt, B. B., \& Vink, M. (2010). On the role of the striatum in response inhibition. PLoS One, 5(11), e13848. doi:10.1371/journal.pone.0013848 
Figure 1.

a)

a)

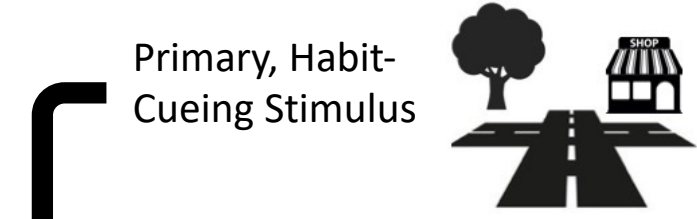

b)

c)

d)

e)

f)

Awareness of unfolding habit

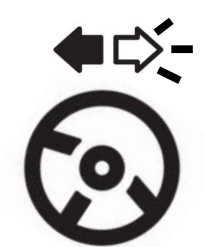

Secondary,

Goal-Relevant

Stimulus

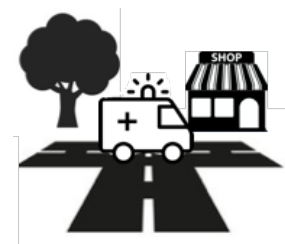

$\rightarrow$

$\rightarrow$

Evaluation of habit

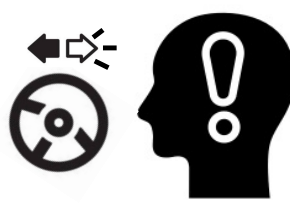
outcome with respect to current goals

\section{Current Goal Habit Outcome}
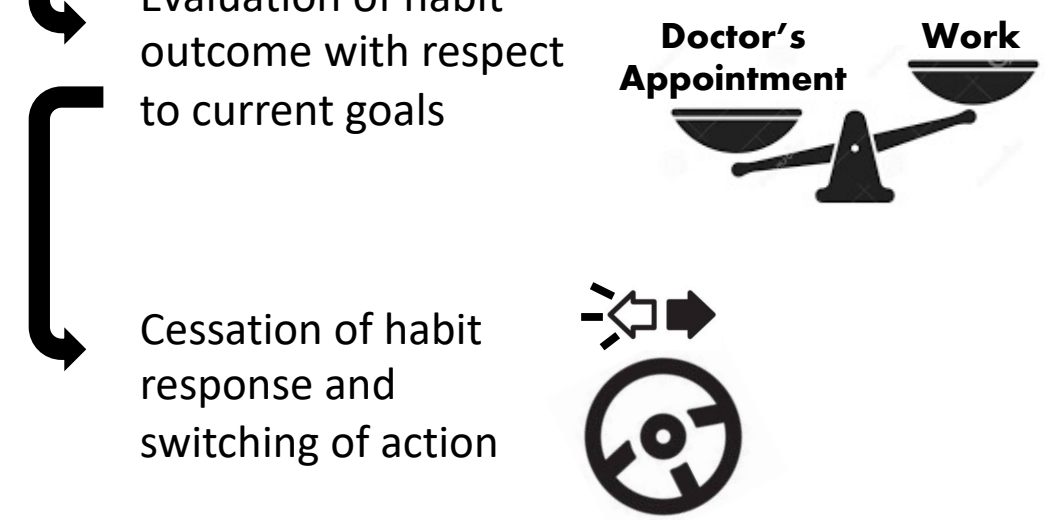

Figure 1. Depiction of the sequence of events involved in "goal-directed stopping" of an unfolding goal-conflicting habit in real time. 
Figure 2.

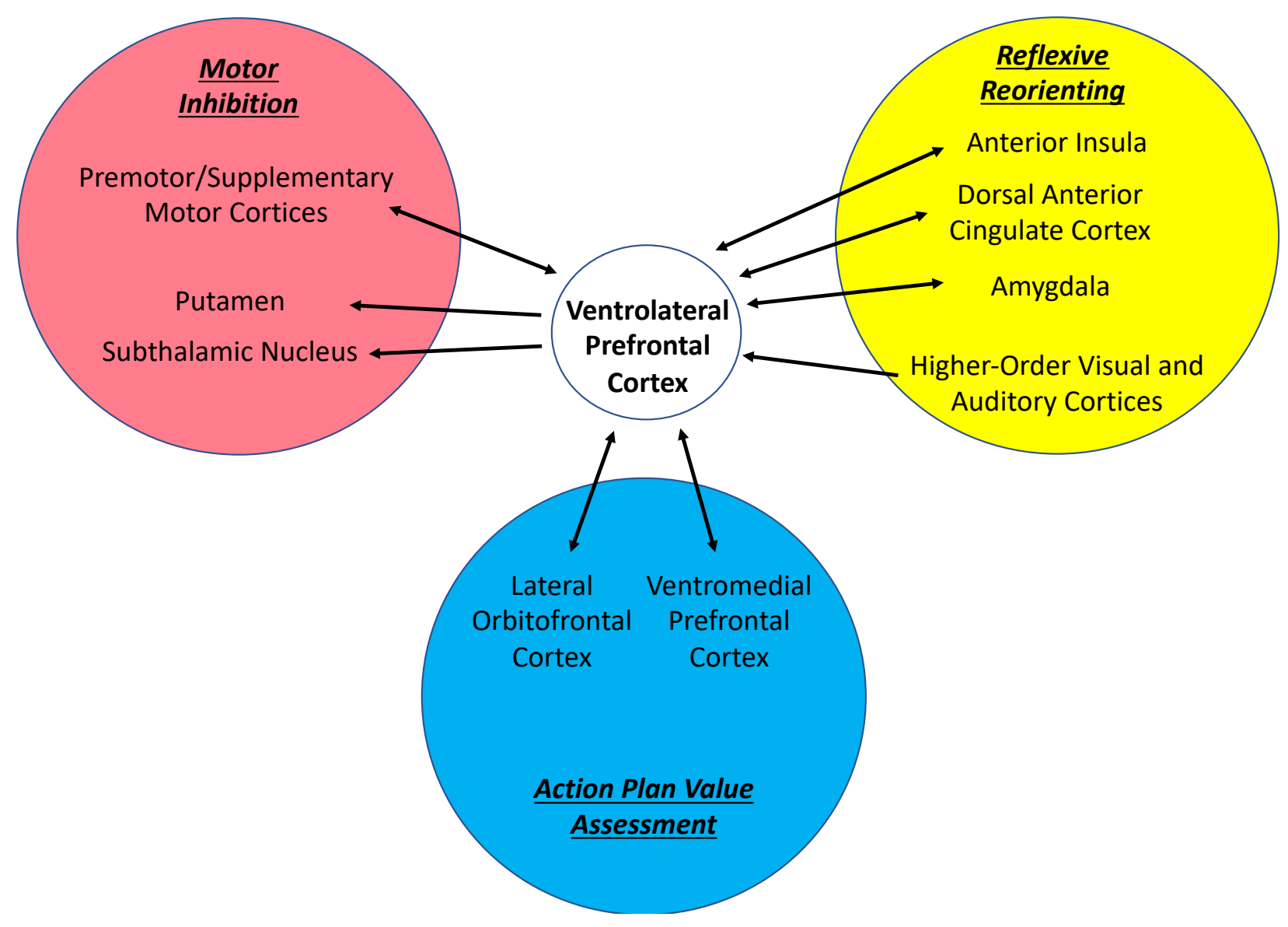

Figure 2. The neural connections of the vlPFC that place it at the nexus of functions - motor inhibition, action plan value assessment, and reflexive reorienting - needed to override the execution of a habit in real time. 